Теорія Ймовір. та Матем. Статист. Вип. 69, 2003
Theor. Probability and Math. Statist.

No. 69, 2004, Pages 85-94 S 0094-9000(05)00616-2

Article electronically published on February 8, 2005

\title{
A CRITERION FOR TESTING HYPOTHESES ABOUT THE COVARIANCE FUNCTION OF A GAUSSIAN STATIONARY PROCESS
}

UDC 519.21

\author{
YU. V. KOZACHENKO AND T. V. FEDORYANYCH
}

\begin{abstract}
New upper and lower bounds for distributions of quadratic forms of Gaussian random variables as well as those for the limits of quadratic forms are found in this paper. Based on these estimates, a criterion is proposed to test a hypothesis about the covariance function $\rho(\tau)$ of a Gaussian stochastic process.
\end{abstract}

\section{INTRODUCTION}

In this paper, we consider the space $S G_{\Xi}(\Omega)$ of square Gaussian random variables and obtain new upper and lower bounds for distributions of quadratic forms of square Gaussian random variables and bounds for distributions of limits of quadratic forms. The upper estimates improve some results of [1, 2].

The inequalities obtained in this paper allow one to construct confidence sets for estimators of the covariance function of a Gaussian stochastic process.

Using these inequalities we propose a criterion to test a hypothesis about the covariance function $\rho(\tau)$ of a Gaussian stochastic process.

\section{The space of square Gaussian random variables}

Definition. Let $\Xi=\{\xi(t), t \in T\}$ be a family of jointly Gaussian random variables (for example, $\xi(t)$ is a Gaussian stochastic process) such that $\mathrm{E} \xi(t)=0$. The set of random variables $\zeta$ that either can be represented in the form

$$
\zeta=\bar{\xi}^{T} A \bar{\eta}-\mathrm{E} \bar{\xi}^{T} A \bar{\eta}
$$

or are the mean square limits of random variables represented in the form of (1) is called the space $S G_{\Xi}(\Omega)$ of square Gaussian random variables; here $\bar{\xi}=\left(\xi_{1}, \ldots, \xi_{d}\right)^{T}$ and $\bar{\eta}=\left(\eta_{1}, \ldots, \eta_{d}\right)^{T}$ are Gaussian random vectors with $\mathrm{E} \bar{\xi}=0$ and $\mathrm{E} \bar{\eta}=0$; the random variables $\xi_{i}, \eta_{i}, i=1, \ldots, d$, belong to $\Xi ; A$ is a symmetric matrix.

It is shown in 1 that

(i) $S G_{\Xi}(\Omega)$ is a Banach space with respect to the norm $\|\zeta\|=\sqrt{\mathrm{E} \zeta^{2}}$;

(ii) $S G_{\Xi}(\Omega)$ is a subspace of the Orlicz space generated by the function

$$
U(x)=\exp \{|x|\}-1 ;
$$

(iii) the norm $\|\zeta\|_{L_{u}(\Omega)}$ on $S G_{\Xi}(\Omega)$ is equivalent to the norm $\sqrt{\mathrm{E} \zeta^{2}}$.

The following result holds for random variables of the space $S G_{\Xi}(\Omega)$.

2000 Mathematics Subject Classification. Primary 60G17; Secondary 60 G07. 
Lemma 2.1 ([1]). Let $\zeta_{i}, i=1,2, \ldots, n$, be random variables of the space $S G_{\Xi}(\Omega)$. Then

$$
\mathrm{E} \exp \left\{\frac{s}{\sqrt{2}} \frac{\zeta}{(\operatorname{Var} \zeta)^{1 / 2}}\right\} \leq R(|s|)
$$

for all $|s|<1$ and all $\lambda_{i} \in \mathbf{R}^{1}, i=1,2, \ldots, n$, where $\zeta=\sum_{i=1}^{n} \lambda_{i} \zeta_{i}$ and

$$
R(s)=\exp \{-s / 2\}(1-s)^{-1 / 2} \text {. }
$$

3. Distributions of QUAdRatic Forms of RANDOM VARIABleS

$$
\text { OF THE SPACE } S G_{\Xi}(\Omega)
$$

The following result improves Lemma 3 in [2].

Lemma 3.1. Let $\bar{\zeta}^{T}=\left(\zeta_{1}, \ldots, \zeta_{d}\right)$ be a random vector such that $\zeta_{i} \in S G_{\Xi}(\Omega)$. Let $A$ be a symmetric positive definite matrix. Then

$$
\mathrm{E} \cosh \left(\sqrt{\frac{t^{2} \bar{\zeta}^{T} A \bar{\zeta}}{\mathrm{E}\left(\bar{\zeta}^{T} A \bar{\zeta}\right)}}\right) \leq R(\sqrt{2} t)
$$

for all $0 \leq t<2^{-1 / 2}$, where $R(t)$ is defined by (2).

Proof. First we consider the case $A=I$, where $I$ is the identity matrix and $\bar{\zeta}$ is such that the random variables $\zeta_{i}$ are orthogonal, that is, $\operatorname{Var}\left(\sum_{i=1}^{d} \lambda_{i} \zeta_{i}\right)=\sum_{i=1}^{d} \lambda_{i}^{2} \mathrm{E} \zeta_{i}^{2}$. Put $\sigma_{i}^{2}=\mathrm{E} \zeta_{i}^{2}, i=1,2, \ldots, d$. It follows from (2) that

$$
\mathrm{E} \exp \left\{\frac{s \sum_{i=1}^{d} \lambda_{i} \zeta_{i}}{\sqrt{2}\left(\sum_{i=1}^{d} \lambda_{i}^{2} \sigma_{i}^{2}\right)^{1 / 2}}\right\} \leq R(|s|)
$$

for all $\lambda_{i} \in \mathbf{R}, i=1,2, \ldots, d$.

Put

$$
u=\frac{s}{\sqrt{2} \sqrt{\sum_{i=1}^{d} \lambda_{i}^{2} \sigma_{i}^{2}}} .
$$

Inequality (4) implies that

$$
\mathrm{E} \exp \left\{u \sum_{i=1}^{d} \lambda_{i} \zeta_{i}\right\} \leq R\left(\sqrt{2}|u| \sqrt{\sum_{i=1}^{d} \lambda_{i}^{2} \sigma_{i}^{2}}\right)
$$

for

$$
|u|<\left(2 \sum_{i=1}^{d} \lambda_{i}^{2} \sigma_{i}^{2}\right)^{-1 / 2}
$$

Put $s_{i}=u \lambda_{i} \sigma_{i}$. Then

$$
\sum_{i=1}^{d} s_{i}^{2}=u^{2} \sum_{i=1}^{d} \lambda_{i}^{2} \sigma_{i}^{2}=\frac{s^{2}}{2}
$$

and $\sum_{i=1}^{d} s_{i}^{2}<\frac{1}{2}$. It follows from (5) that

$$
\mathrm{E} \exp \left\{\sum_{i=1}^{d} s_{i} \frac{\zeta_{i}}{\sigma_{i}}\right\} \leq R\left(\sqrt{2 \sum_{i=1}^{d} s_{i}^{2}}\right)
$$

for all $s_{i}$ such that $\sum_{i=1}^{d} s_{i}^{2}<\frac{1}{2}$. 
Applying inequality (6) we get

$$
\begin{aligned}
\mathrm{E} \prod_{i=1}^{d} \cosh \left(\frac{s_{i} \zeta_{i}}{\sigma_{i}}\right) & =\mathrm{E} \prod_{i=1}^{d} \frac{\exp \left\{s_{i} \zeta_{i} / \sigma_{i}\right\}+\exp \left\{-s_{i} \zeta_{i} / \sigma_{i}\right\}}{2} \\
& =\frac{1}{2^{d}} \mathrm{E} \prod_{i=1}^{d}\left(\exp \left\{\frac{s_{i} \zeta_{i}}{\sigma_{i}}\right\}+\exp \left\{-\frac{s_{i} \zeta_{i}}{\sigma_{i}}\right\}\right) \\
& =\frac{1}{2^{d}} \sum \mathrm{E} \prod_{i=1}^{d} \exp \left\{\frac{s_{i} \zeta_{i} \delta_{i}}{\sigma_{i}}\right\}=\frac{1}{2^{d}} \sum \mathrm{E} \exp \left\{\sum_{i=1}^{d} \frac{s_{i} \zeta_{i} \delta_{i}}{\sigma_{i}}\right\} \\
& \leq \frac{1}{2^{d}} \sum R\left(\sqrt{2 \sum_{i=1}^{d} s_{i}^{2}}\right)=R\left(\sqrt{2 \sum_{i=1}^{d} s_{i}^{2}}\right)
\end{aligned}
$$

where $\delta_{i}= \pm 1$. Therefore

$$
\mathrm{E} \prod_{i=1}^{d} \cosh \left(\frac{s_{i} \zeta_{i}}{\sigma_{i}}\right) \leq R\left(\sqrt{2 \sum_{i=1}^{d} s_{i}^{2}}\right) .
$$

Put $f(z)=\ln \cosh \sqrt{z}, z>0$. The function $f(z)$ is concave since $f(0)=\ln \cosh 0=0$ and $f^{\prime \prime}(z)<0$. Thus

$$
\sum_{i=1}^{d} f\left(z_{i}\right) \geq f\left(\sum_{i=1}^{d} z_{i}\right)
$$

for all $z_{i}>0, i=1,2, \ldots, d$. This means that

$$
\prod_{i=1}^{d} \cosh \sqrt{z_{i}} \geq \cosh \sqrt{\sum_{i=1}^{d} z_{i},} \quad z_{i}>0 .
$$

Therefore

$$
\begin{aligned}
\mathrm{E} \cosh \sqrt{\sum_{i=1}^{d} \frac{s_{i}^{2} \zeta_{i}^{2}}{\sigma_{i}^{2}}} & \leq \mathrm{E} \prod_{i=1}^{d} \cosh \sqrt{\frac{s_{i}^{2} \zeta_{i}^{2}}{\sigma_{i}^{2}}}=\mathrm{E} \prod_{i=1}^{d} \cosh \left\{\frac{\left|s_{i} \zeta_{i}\right|}{\sigma_{i}}\right\}=\mathrm{E} \prod_{i=1}^{d} \cosh \left\{\frac{s_{i} \zeta_{i}}{\sigma_{i}}\right\} \\
& \leq R\left(\sqrt{2 \sum_{i=1}^{d} s_{i}^{2}}\right)
\end{aligned}
$$

if $\sum_{i=1}^{d} s_{i}^{2}<\frac{1}{2}$. Set

$$
s_{i}^{2}=\frac{\sigma_{i}^{2} t^{2}}{\sum_{i=1}^{d} \sigma_{i}^{2}} .
$$

Then the latter inequality implies that

$$
\mathrm{E} \cosh \left(\sqrt{\frac{t^{2} \sum_{i=1}^{d} \zeta_{i}^{2}}{\sum_{i=1}^{d} \sigma_{i}^{2}}}\right) \leq R(\sqrt{2} t)
$$

for $0 \leq t<2^{-1 / 2}$.

Now we turn to the general case. Let $B$ be a symmetric matrix such that $B B^{T}=$ $B^{2}=A$. Let $R=\operatorname{cov} \bar{\zeta}$, and let $O$ be the orthogonal matrix that reduces $B R B$ to the diagonal form, namely

$$
O B R B O^{T}=D=\operatorname{diag}\left(d_{k}^{2}\right)_{k=1}^{d} .
$$


Let $\bar{\theta}=O B \bar{\zeta}$. Then

$$
\bar{\theta}^{T} \bar{\theta}=\bar{\zeta}^{T} B O^{T} O B \bar{\zeta}=\bar{\zeta}^{T} A \bar{\zeta}
$$

and $\operatorname{cov} \bar{\theta}=O B \operatorname{cov} \zeta B O^{T}=D$. Since $\theta_{i} \in S G_{\Xi}(\Omega)$, inequality (7) holds for $\bar{\theta}$ instead of $\zeta$ where $\bar{\theta}^{T}=\left(\theta_{1}, \ldots, \theta_{d}\right)$. Since

$$
\bar{\theta}^{T} \bar{\theta}=\sum_{i=1}^{d} \theta_{i}^{2}=\bar{\zeta}^{T} A \bar{\zeta}
$$

we get

$$
\cosh \sqrt{\frac{t^{2} \bar{\theta}^{T} \bar{\theta}}{\mathrm{E} \bar{\theta}^{T} \bar{\theta}}}=\cosh \sqrt{\frac{t^{2} \bar{\zeta}^{T} A \bar{\zeta}}{\mathrm{E} \bar{\zeta}^{T} A \bar{\zeta}}}
$$

The lemma is proved.

Corollary 3.1. Let the assumptions of Lemma 3.1 hold. If

$$
\eta=\underset{n \rightarrow \infty}{\operatorname{li} . \operatorname{m}} \bar{\zeta}_{n}^{T} A_{n} \bar{\zeta}_{n}
$$

then

$$
\mathrm{E} \cosh \left(\sqrt{\frac{t^{2} \eta}{\mathrm{E} \eta}}\right) \leq R(\sqrt{2} t)
$$

Corollary 3.1 follows from the Fatou lemma.

Lemma 3.2. Let the assumptions of Lemma 3.1 hold. Then

$$
\mathrm{P}\left\{\frac{\eta}{\mathrm{E} \eta}>x\right\} \leq \frac{2^{1 / 4} x^{1 / 4}}{\cosh \left(\sqrt{x / 2}-\frac{1}{2}\right)}
$$

for $x>\frac{1}{2}$ where either $\eta=\bar{\zeta}_{n}^{T} A_{n} \bar{\zeta}_{n}$ or $\eta=$ l. i. m. $_{n \rightarrow \infty} \bar{\zeta}_{n}^{T} A_{n} \bar{\zeta}_{n}$.

Proof. It follows from the Chebyshev inequality and (3) in the case of $\eta=\bar{\zeta}_{n}^{T} A_{n} \bar{\zeta}_{n}$, or from Corollary 3.1 in the case of $\eta=1$. i. m. $_{n \rightarrow \infty} \bar{\zeta}_{n}^{T} A_{n} \bar{\zeta}_{n}$, that

$$
\mathrm{P}\left\{\frac{\eta}{\mathrm{E} \eta}>x\right\} \leq \frac{\mathrm{E} \cosh \sqrt{t^{2} \eta / \mathrm{E} \eta}}{\cosh \sqrt{t^{2} x}} \leq \frac{R(\sqrt{2} t)}{\cosh \sqrt{t^{2} x}}
$$

for $x>0$ and $0 \leq t<2^{-1 / 2}$. Put $t=2^{-1 / 2}-\left(2 x^{1 / 2}\right)^{-1}$ for $x>\frac{1}{2}$. Then

$$
\frac{R(\sqrt{2} t)}{\cosh \sqrt{t^{2} x}}=\frac{(2 x)^{1 / 4} \exp \left\{(2 \sqrt{2 x})^{-1}-\frac{1}{2}\right\}}{\cosh \left(\sqrt{x / 2}-\frac{1}{2}\right)} .
$$

Since $\exp \left\{\left(2(2 x)^{1 / 2}\right)^{-1}-\frac{1}{2}\right\}<1$ for $x>\frac{1}{2}$, we get

$$
\mathrm{P}\left\{\frac{\eta}{\mathrm{E} \eta}>x\right\} \leq \frac{2^{1 / 4} x^{1 / 4}}{\cosh \left(\sqrt{x / 2}-\frac{1}{2}\right)} .
$$

Lemma 3.2 is proved.

Lemma 3.3. Let $\xi_{1}, \xi_{2}, \ldots, \xi_{m}$ be independent normal random variables such that

$$
\mathrm{E} \xi_{k}=0 \quad \text { and } \quad \mathrm{E} \xi_{k}^{2}=\sigma_{k}^{2},
$$

and let $c_{k}= \pm 1$ and $s>0$. Then

$$
\left|\operatorname{Eexp}\left\{i \frac{s \sum_{k=1}^{m} \xi_{k}^{2} c_{k}}{2\left(\sum_{k=1}^{m} \sigma_{k}^{4}\right)^{1 / 2}}\right\}\right| \leq \frac{1}{\left(1+s^{2}\right)^{1 / 4}} .
$$


Proof. We have

$$
\mathrm{E} \exp \left\{i \frac{\sum_{k=1}^{m} \xi_{k}^{2} c_{k}}{r}\right\}=\mathrm{E} \prod_{k=1}^{m} \exp \left\{\frac{i \xi_{k}^{2} c_{k}}{r}\right\} .
$$

Taking the equality

$$
\mathrm{E} \exp \left\{i s \xi_{k}^{2}\right\}=\left(1-2 i s \sigma_{k}^{2}\right)^{-1 / 2}
$$

into account, we rewrite (9) in the following form:

$$
\mathrm{E} \exp \left\{i \frac{\sum_{k=1}^{m} \xi_{k}^{2} c_{k}}{r}\right\}=\prod_{k=1}^{m}\left(1-2 i \frac{\sigma_{k}^{2} c_{k}}{r}\right)^{-1 / 2}
$$

Thus

$$
\begin{aligned}
\left|\mathrm{E} \exp \left\{i \frac{\sum_{k=1}^{m} \xi_{k}^{2} c_{k}}{r}\right\}\right| & =\left|\prod_{k=1}^{m}\left(1-2 i \frac{\sigma_{k}^{2} c_{k}}{r}\right)^{-1 / 2}\right|=\prod_{k=1}^{m}\left|1-2 i \frac{\sigma_{k}^{2} c_{k}}{r}\right|^{-1 / 2} \\
& =\prod_{k=1}^{m}\left(1+\left(2 \frac{\sigma_{k}^{2} c_{k}}{r}\right)^{2}\right)^{-1 / 4}=\prod_{k=1}^{m}\left(1+\frac{4 \sigma_{k}^{4}}{r^{2}}\right)^{-1 / 4}
\end{aligned}
$$

Put

$$
I=\prod_{k=1}^{m}\left(1+\frac{4 \sigma_{k}^{4}}{r^{2}}\right)^{-1 / 4}
$$

Then

$$
\ln I=-\frac{1}{4} \sum_{k=1}^{m} \ln \left(1+\frac{4 \sigma_{k}^{4}}{r^{2}}\right) .
$$

Consider the function $f(x)=\ln (1+x)$ for $x>0$. It is clear that $f(0)=0, f(x)$ is concave, and thus

$$
f\left(\sum_{k=1}^{m} x_{k}\right) \leq \sum_{k=1}^{m} f\left(x_{k}\right), \quad x_{k} \geq 0 .
$$

Furthermore

$$
-\sum_{k=1}^{m} f\left(x_{k}\right) \leq-f\left(\sum_{k=1}^{m} x_{k}\right)
$$

whence

$$
\ln I \leq-\frac{1}{4} \ln \left(1+\frac{4}{r^{2}} \sum_{k=1}^{m} \sigma_{k}^{4}\right)
$$

and

$$
I \leq\left(1+\frac{4}{r^{2}} \sum_{k=1}^{m} \sigma_{k}^{4}\right)^{-1 / 4}
$$

in view of (10).

Let

$$
r=\frac{2\left(\sum_{k=1}^{m} \sigma_{k}^{4}\right)^{1 / 2}}{s}, \quad s>0 .
$$

Then $I \leq\left(1+s^{2}\right)^{-1 / 4}$ for $s>0$.

Lemma 3.3 is proved. 
Theorem 3.1. Let $A$ be a symmetric matrix and $\bar{\xi}^{T}=\left(\xi_{1}, \xi_{2}, \ldots, \xi_{m}\right)$ a random vector whose coordinates $\xi_{k}$ are normal random variables such that $\mathrm{E} \xi_{k}=0$ and $\mathrm{E} \xi_{k}^{2}=\sigma_{k}^{2}$. Then

$$
\left|\mathrm{E} \exp \left\{i \frac{s\left(\bar{\xi}^{T} A \bar{\xi}-\mathrm{E} \bar{\xi}^{T} A \bar{\xi}\right)}{\sqrt{2} \sqrt{D \bar{\xi}^{T} A \bar{\xi}}}\right\}\right| \leq \frac{1}{\left(1+s^{2}\right)^{1 / 4}}, \quad s>0 .
$$

Theorem 3.1 easily follows from Lemma 3.3.

Lemma 3.4. Let $\bar{\zeta}^{T}=\left(\zeta_{1}, \ldots, \zeta_{n}\right)$ be a random vector whose coordinates $\zeta_{j}, 1 \leq j \leq n$, belong to the space $S G_{\Xi}(\Omega)$. Let $A$ be a symmetric positive definite matrix. Then

$$
\mathrm{E} \exp \left\{-\frac{u^{2}}{2} \frac{\bar{\zeta}^{T} A \bar{\zeta}}{\mathrm{E} \bar{\zeta}^{T} A \bar{\zeta}}\right\} \leq g(u)
$$

where

$$
g(u)=\frac{1}{\sqrt{2 \pi}} \int_{-\infty}^{+\infty} \exp \left\{-\frac{s^{2}}{2}\right\} \frac{d s}{\left(1+u^{2} s^{2}\right)^{1 / 4}}, \quad 0<u<1 .
$$

Proof. First let $\zeta_{j}$ be orthogonal square Gaussian random variables such that $\mathrm{E} \zeta_{j}^{2}=\sigma_{j}^{2}$ and $\sigma_{j}^{2}>0$. Let $\lambda_{j} \in \mathbf{R}^{1}$. Then Lemma 3.1 and the Fatou lemma imply

$$
\left|\mathrm{E} \exp \left\{i \frac{s}{\sqrt{2}} \frac{\sum_{j=1}^{n} \lambda_{j} \zeta_{j}}{\left(\sum_{j=1}^{n} \lambda_{j}^{2} \sigma_{j}^{2}\right)^{1 / 2}}\right\}\right| \leq \frac{1}{\left(1+s^{2}\right)^{1 / 4}} .
$$

We rewrite the left-hand side of (11) in the following form:

$$
\left|\mathrm{E} \exp \left\{i \frac{s}{\sqrt{2}} \frac{\sum_{j=1}^{n} \frac{\zeta_{j}}{\sigma_{j}}\left(\lambda_{j} \sigma_{j}\right)}{\left(\sum_{j=1}^{n} \lambda_{j}^{2} \sigma_{j}^{2}\right)^{1 / 2}}\right\}\right| .
$$

Put

$$
s_{j}=s \frac{\lambda_{j} \sigma_{j}}{\left(\sum_{j=1}^{n} \lambda_{j}^{2} \sigma_{j}^{2}\right)^{1 / 2}}, \quad s^{2}=\sum_{j=1}^{n} s_{j}^{2} .
$$

Then (11) can be rewritten in the following form:

$$
\left|\mathrm{E} \exp \left\{i \frac{1}{\sqrt{2}} \sum_{j=1}^{n} \frac{s_{j} \zeta_{j}}{\sigma_{j}}\right\}\right| \leq \frac{1}{\left(1+\sum_{j=1}^{n} s_{j}^{2}\right)^{1 / 4}} .
$$

For $t_{j}>0$ we have

$$
\begin{aligned}
\int_{\mathbf{R}^{n}} & \ldots \int \mathrm{E} \exp \left\{i \frac{1}{\sqrt{2}} \sum_{j=1}^{n} s_{j} \frac{\zeta_{j}}{\sigma_{j}}\right\} \prod_{j=1}^{n} \frac{1}{\sqrt{2 \pi} t_{j}} \exp \left\{-\frac{s_{j}^{2}}{2 t_{j}^{2}}\right\} d s_{1} \cdots d s_{n} \\
& =\mathrm{E} \exp \left\{-\sum_{j=1}^{n} \frac{\zeta_{j}^{2} t_{j}^{2}}{2 \sigma_{j}^{2}}\right\},
\end{aligned}
$$

whence

$$
\mathrm{E} \exp \left\{-\sum_{j=1}^{n} \frac{\zeta_{j}^{2} t_{j}^{2}}{2 \sigma_{j}^{2}}\right\} \leq \int_{\mathbf{R}^{n}} \ldots \int \prod_{j=1}^{n}\left(\frac{1}{\sqrt{2 \pi} t_{j}}\right) \exp \left\{-\frac{s_{j}^{2}}{2 t_{j}^{2}}\right\} \frac{d s_{1} \cdots d s_{n}}{\left(1+\sum_{j=1}^{n} s_{j}^{2}\right)^{1 / 4}}
$$


in view of inequality (12). Setting $s_{j} / t_{j}=u_{j}$ we obtain

$$
\begin{aligned}
\mathrm{E} \exp & \left\{-\sum_{j=1}^{n} \frac{\zeta_{j}^{2} t_{j}^{2}}{2 \sigma_{j}^{2}}\right\} \\
& \leq \int_{\mathbf{R}^{n}} \ldots \int\left(\frac{1}{\sqrt{2 \pi}}\right)^{n} \exp \left\{-\frac{1}{2} \sum_{j=1}^{n} u_{j}^{2}\right\} \frac{d u_{1} \cdots d u_{n}}{\left(1+\sum_{j=1}^{n} t_{j}^{2} u_{j}^{2}\right)^{1 / 4}} .
\end{aligned}
$$

Put

$$
t_{j}^{2}=\sigma_{j}^{2} \frac{u^{2}}{\sum_{j=1}^{n} \sigma_{j}^{2}}, \quad \sum_{j=1}^{n} t_{j}^{2}=u^{2} .
$$

Since the function $f(u)=\frac{1}{4} \ln (1+u)$ is concave and $f(0)=0$, we have

$$
\frac{1}{4} \ln \left(1+\sum_{i=1}^{n} \alpha_{i} x_{i}\right) \geq \sum_{i=1}^{n} \alpha_{i}\left(\frac{1}{4} \ln \left(1+x_{i}\right)\right)
$$

for $\alpha_{i}>0$ such that $\sum_{i=1}^{n} \alpha_{i}=1$. Further

$$
-\frac{1}{4} \ln \left(1+\sum_{i=1}^{n} \alpha_{i} x_{i}\right) \leq \sum_{i=1}^{n} \alpha_{i}\left(-\frac{1}{4} \ln \left(1+x_{i}\right)\right) .
$$

Thus

$$
\frac{1}{\left(1+\sum_{i=1}^{n} \alpha_{i} x_{i}\right)^{1 / 4}} \leq \prod_{i=1}^{n} \frac{1}{\left(1+x_{i}\right)^{(1 / 4)^{\alpha_{i}}}} .
$$

Therefore

$$
\frac{1}{\left(1+\sum_{i=1}^{n} t_{j}^{2} u_{j}^{2}\right)^{1 / 4}}=\frac{1}{\left(1+\sum_{i=1}^{n} \frac{t_{j}^{2}}{u^{2}} u_{j}^{2} u^{2}\right)^{1 / 4}} \leq \prod_{j=1}^{n} \frac{1}{\left(1+u_{j}^{2} u^{2}\right)^{t_{j}^{2} /\left(4 u^{2}\right)}},
$$

since $\sum_{j=1}^{n} t_{j}^{2} / u^{2}=1$. This inequality together with (13) yields

$$
\mathrm{E} \exp \left\{-\sum_{j=1}^{n} \frac{\zeta_{j}^{2} t_{j}^{2}}{2 \sigma_{j}^{2}}\right\} \leq \prod_{j=1}^{n} \mathrm{E}\left(\left(\frac{1}{\left(1+\xi_{j}^{2} u^{2}\right)^{1 / 4}}\right)^{t_{j}^{2} / u^{2}}\right)
$$

where $\xi_{j}$ are Gaussian $N(0,1)$ independent random variables. Applying the inequality $\mathrm{E}|\xi|^{\alpha} \leq(\mathrm{E}|\xi|)^{\alpha}, \alpha<1$, we obtain

$$
\begin{aligned}
\mathrm{E} \exp \left\{-\sum_{j=1}^{n} \frac{\zeta_{j}^{2} t_{j}^{2}}{2 \sigma_{j}^{2}}\right\} & \leq\left(\mathrm{E}\left(\frac{1}{\left(1+\xi^{2} u^{2}\right)^{1 / 4}}\right)\right)^{\sum_{j=1}^{n} t_{j}^{2} / u^{2}} \\
& =\frac{1}{\sqrt{2 \pi}} \int_{-\infty}^{+\infty} \exp \left\{-\frac{s^{2}}{2}\right\} \frac{d s}{\left(1+s^{2} u^{2}\right)^{1 / 4}}=g(u) .
\end{aligned}
$$

Therefore

$$
\mathrm{E} \exp \left\{-\frac{u^{2}}{2} \frac{\sum_{j=1}^{n} \zeta_{j}^{2}}{\sum_{j=1}^{n} \sigma_{j}^{2}}\right\} \leq g(u)
$$

where

$$
g(u)=\frac{1}{\sqrt{2 \pi}} \int_{-\infty}^{+\infty} \exp \left\{-\frac{s^{2}}{2}\right\} \frac{d s}{\left(1+s^{2} u^{2}\right)^{1 / 4}} .
$$


Now we consider the general case. Let $B$ be a matrix such that $B B^{T}=B^{2}=A$, and let $R=\operatorname{cov} \bar{\zeta}$. Let $O$ be the orthogonal matrix that reduces the matrix $B R B$ to the diagonal form, namely

$$
O B R B O^{T}=D=\operatorname{diag}\left(d_{k}^{2}\right)_{k=1}^{n} .
$$

Putting $\bar{\theta}=O B \bar{\zeta}$ we get

$$
\bar{\theta}^{T} \bar{\theta}=\bar{\zeta}^{T} B O^{T} O B \bar{\zeta}=\bar{\zeta}^{T} A \bar{\zeta}
$$

and $\operatorname{cov} \bar{\theta}=O B \operatorname{cov} \bar{\zeta} B O^{T}=D$. Since $\theta_{i} \in S G_{\Xi}(\Omega)$ and $\bar{\theta}^{T}=\left(\theta_{1}, \ldots, \theta_{n}\right)$, the lemma holds for $\bar{\theta}$ instead of $\zeta$. Thus

$$
\bar{\theta}^{T} \bar{\theta}=\sum_{i=1}^{n} \theta_{i}^{2}=\bar{\zeta}^{T} A \bar{\zeta}
$$

and

$$
\mathrm{E} \exp \left\{-\frac{u^{2}}{2} \frac{\bar{\zeta}^{T} A \bar{\zeta}}{\mathrm{E} \bar{\zeta}^{T} A \bar{\zeta}}\right\} \leq g(u)
$$

The lemma is proved.

Theorem 3.2. Let the assumptions of Lemmas 3.2 and 3.4 hold. Then

$$
\mathrm{P}\left\{\frac{\eta}{\mathrm{E} \eta}>x\right\} \geq 1-g(u) \exp \left\{\frac{u^{2} x}{2}\right\}
$$

for all $1>u>0$ and $x<-2 \ln g(u) / u^{2}$. Moreover

$$
\mathrm{P}\left\{\frac{\eta}{\mathrm{E} \eta}>x\right\} \leq \frac{2^{1 / 4} x^{1 / 4}}{\cosh \left(\sqrt{x / 2}-\frac{1}{2}\right)}
$$

for $x>\frac{1}{2}$ where

$$
g(u)=\frac{1}{\sqrt{2 \pi}} \int_{-\infty}^{+\infty} \exp \left\{-\frac{s^{2}}{2}\right\} \frac{d s}{\left(1+s^{2} u^{2}\right)^{1 / 4}}
$$

and either $\eta=\bar{\zeta}_{n}^{T} A_{n} \bar{\zeta}_{n}$ or $\eta=$ l. i. m. $\bar{\zeta}_{n}^{T} A_{n} \bar{\zeta}_{n}, \bar{\zeta}^{T}=\left(\zeta_{1}, \ldots, \zeta_{d}\right), \zeta_{i} \in S G_{\Xi}(\Omega)$.

Proof. According to Lemma 3.4

$$
\mathrm{E} \exp \left\{-\frac{u^{2} \eta}{2 \mathrm{E} \eta}\right\} \leq g(u)
$$

Put $\theta=\eta / \mathrm{E} \eta$. Then

$$
\begin{aligned}
\mathrm{P}\{\theta<x\} & =\int_{0}^{x} d F(v)=\int_{0}^{x} \frac{\exp \left\{-u^{2} v / 2\right\}}{\exp \left\{-u^{2} v / 2\right\}} d F(v) \leq \frac{1}{\exp \left\{-u^{2} x / 2\right\}} \mathrm{E} \exp \left\{-\frac{u^{2} \theta}{2}\right\} \\
& \leq \frac{g(u)}{\exp \left\{-u^{2} x / 2\right\}}=g(u) \exp \left\{\frac{u^{2} x}{2}\right\},
\end{aligned}
$$

whence

$$
\mathrm{P}\{\theta>x\} \geq 1-g(u) \exp \left\{\frac{u^{2} x}{2}\right\} .
$$

Using the initial notation we have

$$
\mathrm{P}\left\{\frac{\eta}{\mathrm{E} \eta}>x\right\} \geq 1-g(u) \exp \left\{\frac{u^{2} x}{2}\right\} .
$$

Inequality (15) is proved in Lemma 3.2. 


\section{The CONSTRUCtion OF A CRITERION TO TEST HyPOTHESES}

Let $\xi(t)$ be a Gaussian stochastic process with $\mathrm{E} \xi(t)=0$ and the correlation function

$$
\mathrm{E} \xi(t+\tau) \xi(t)=\rho(\tau)
$$

Let

$$
\hat{\rho}(\tau)=\frac{1}{T} \int_{0}^{T} \xi(t+\tau) \xi(t) d t, \quad 0 \leq \tau \leq T,
$$

be an estimator of the covariance function $\rho(\tau)$. Put $\chi(\tau)=\hat{\rho}(\tau)-\rho(\tau)$. It is obvious that $\chi(\tau)$ is a square Gaussian random variable.

Further let

$$
\eta=\int_{0}^{B}(\hat{\rho}(\tau)-\rho(\tau))^{2} d \tau
$$

It is obvious that inequalities (14)-(15) hold for $\eta$ where

$$
\begin{aligned}
\mathrm{E} \eta & =\mathrm{E} \int_{0}^{B}(\hat{\rho}(\tau)-\rho(\tau))^{2} d \tau \\
& =\frac{1}{T^{2}} \int_{0}^{B} \int_{0}^{T} \int_{0}^{T}\left[\rho^{2}(t-s)+\rho(t+\tau-s) \rho(t-\tau-s)\right] d t d s d \tau .
\end{aligned}
$$

Put

$$
f(x)=\frac{2^{1 / 4} x^{1 / 4}}{\cosh \left(\sqrt{x / 2}-\frac{1}{2}\right)}, \quad s(x, u)=1-g(u) \exp \left\{\frac{u^{2} x}{2}\right\}
$$

for $g(u)$ defined as in Theorem 3.2.

It follows from (14) and (15) that

$$
\mathrm{P}\left\{\frac{\eta}{\mathrm{E} \eta} \notin[x ; y]\right\} \leq 1-s(x, u)+f(y)
$$

whence

$$
\mathrm{P}\left\{\frac{\eta}{\mathrm{E} \eta} \in[x ; y]\right\} \geq s(x, u)-f(y)
$$

for $1>u>0, x<-2 \ln g(u) / u^{2}$, and $y>\frac{1}{2}$.

Let $H$ be the hypothesis that the covariance function of a Gaussian stochastic process equals $\rho(\tau)$ for $0 \leq \tau \leq T$. We regard $\hat{\rho}(\tau)$ as the estimator of the function $\rho(\tau)$.

To test the hypothesis $H$ one can use the following criterion.

Criterion. Given $\alpha, 0<\alpha<1$, one should determine $x_{\alpha}$ and $y_{\alpha}$ such that

$$
1-s\left(x_{\alpha}, u\right)+f\left(y_{\alpha}\right)=\alpha .
$$

The hypothesis $H$ is accepted if

$$
x_{\alpha}<\frac{\int_{0}^{B}(\hat{\rho}(\tau)-\rho(\tau))^{2} d \tau}{\mathrm{E} \int_{0}^{B}(\hat{\rho}(\tau)-\rho(\tau))^{2} d \tau}<y_{\alpha} .
$$

Otherwise the hypothesis is rejected.

We now show how to find $a$ and $b$ such that

$$
\mathrm{P}\left\{a \leq \frac{\eta}{\mathrm{E} \eta} \leq b\right\} \geq 1-\alpha
$$

if $\alpha$ is given. In other words we want to find $a$ and $b$ such that

$$
\mathrm{P}\left\{\frac{\eta}{\mathrm{E} \eta} \notin[a ; b]\right\} \leq \alpha
$$


if $\alpha$ is given. The latter inequality follows from

$$
\mathrm{P}\left\{\frac{\eta}{\mathrm{E} \eta} \leq a\right\} \leq \alpha \gamma \quad \text { and } \quad \mathrm{P}\left\{\frac{\eta}{\mathrm{E} \eta} \geq b\right\} \leq \alpha(1-\gamma)
$$

for some $0<\gamma<1$.

We choose the constant $\gamma$ to minimize the difference $b_{\gamma}-a_{\gamma}$ where $a_{\gamma}$ and $b_{\gamma}$ are solutions of the equations

$$
\begin{gathered}
g(u) \exp \left\{\frac{u^{2} a}{2}\right\}=\alpha \gamma, \\
\frac{2^{1 / 4} b^{1 / 4}}{\cosh \left(\sqrt{b / 2}-\frac{1}{2}\right)}=\alpha(1-\gamma) .
\end{gathered}
$$

\section{BIBLIOGRAPHY}

1. V. V. Buldygin and Yu. V. Kozachenko, Metric Characterization of Random Variables and Random Processes, TViMS, Kiev, 1998; English transl., AMS, Providence, 2000. MR1743716 (2001g:60089)

2. Yu. V. Kozachenko and O. V. Stus, Square Gaussian random processes and estimator of covariance functions, Math. Commun. 3 (1998), no. 1, 83-94. MR 1648867 (2000b:60099)

3. M. A. Krasnosel'skiı̌ and Ya. B. Rutickiı̌, Convex Functions and Orlicz Spaces, Fizmatgiz, Moscow, 1958; English transl., Noordhof, Gröningen, 1961. MR0106412 (21:5144)

Department of Probability Theory and Mathematical Statistics, Faculty for Mechanics and Mathematics, Kyiv National Taras Shevchenko University, Glushkova 6, Kyiv 03127, UKRAINE

E-mail address: yvkuniv.kiev.ua

Department of Probability Theory and Mathematical Statistics, Faculty for Mechanics and Mathematics, Kyiv National Taras Shevchenko University, Glushkova 6, Kyiv 03127, UKRAINE

E-mail address: fedoryanichuniv.kiev.ua

Received 19/DEC/2002

Translated by OLEG KLESOV 